\title{
Optimization of chromatography method and analytical conditions for isotope analyses of $\mathrm{Li}$ via MC-ICP- \\ MS
}

\author{
XIAOQIANG $\operatorname{Li}^{1}$, GUILIN HAN ${ }^{1 *}$
}

${ }^{1}$ Institute of Earth Sciences, China University of Geosciences (Beijing), Beijing, 100083, China (* correspondence:

hanguiling@cugb.edu.cn, xiaoqli@cugb.edu.cn)

The lithium isotope is an effective silicate weathering proxy because of high content in silicates and low content in carbonates. However, complex matrix extraction conditions limit the accurate and precise determination of lithium via MC-ICP-MS. Here, a new separation method using dual ionexchange column permits the complete purification of $\mathrm{Li}$ from matrix element. The advantage of this method is that it achieves a high yield of $\mathrm{Li}$ under complex matrix extraction conditions. To provide an optimized method for achieving high accurate and precise determination of $\mathrm{Li}$ isotope, we systematically assess the effect of matrix element, acid strength and concentration mismatch on the acquire accurate and precise analysis of Li isotopes in 'dry' plasma and 'wet' plasma conditions. Complete separation and strict matching of acid strength and concentration are critical because of distinct deviations and mass-independent fractionations. Therefore, acid strength and concentrations of samples and standards were matched to within $\pm 5 \%$ of each other in this study. Overall, the long-term external precision was better than $0.3-0.4 \%$ (2SD) that reported in most laboratory around the world. Reference rock materials including BHVO-2, BCR-2, AGV-2, GSP-2, RGM-2, JG-2, SGR-1b and IAPSO (seawater) were digested multiple times to further evaluate the precision and accuracy of lithium isotope. Their $\delta^{7} \mathrm{Li}$ are consistent with the previously published data. These results show that our optimized method is efficient and the data obtained are reliable. 